Meta

Journal des traducteurs

Translators' Journal

\title{
Regards sur la terminologisation en lexicologie
}

\section{Yves Gentilhomme-Koutyrine}

Volume 39, numéro 4, décembre 1994

Hommage à Bernard Quemada : termes et textes

URI : https://id.erudit.org/iderudit/002880ar

DOI : https://doi.org/10.7202/002880ar

Aller au sommaire du numéro

Éditeur(s)

Les Presses de l'Université de Montréal

ISSN

0026-0452 (imprimé)

1492-1421 (numérique)

Découvrir la revue

Citer cet article

Gentilhomme-Koutyrine, Y. (1994). Regards sur la terminologisation en lexicologie. Meta, 39(4), 546-560. https://doi.org/10.7202/002880ar

\section{Résumé de l'article}

Chaque époque est imprégnée par certaines idées générales ("Paradigmes" selon Th. S. Kuhn) sur ce qu'est la science, la rigueur, la vocation, la formulation des résultats obtenus... Le $D E C$ fait écho à cette quête d'idéal. Le présent article se limite à attirer l'attention sur quelques retombées de cette initiative invitant à des recherches plus poussées sur l'opposition lexie (lexeme) / terme et suggère la création de dictionnaires technolinguistiques appropriés. 


\title{
REGARDS SUR LA TERMINOLOGISATION EN LEXICOLOGIE
}

\author{
YVES GENTILHOMME-KOUTYRINE
}

Besançon, France

\begin{abstract}
Résumé
Chaque époque est imprégnée par certaines idées générales ( Paradigmes» selon $T h$. S. Kuhn) sur ce qu'est la science, la rigueur, la vocation, la formulation des résultats obtenus... Le DEC fait écho à cette quête d'idéal. Le présent article se limite à attirer l'attention sur quelques retombées de cette initiative invitant à des recherches plus poussées sur l'opposition lexie (lexème) / terme et suggère la création de dictionnaires technolinguistiques appropriés.
\end{abstract}

\begin{abstract}
Each period in time is permeated by certain general ideas (Paradigms, Th. S. Kuhn) concerning the definition of science, rigour, vocation, formulation of results... The Dictionnaire explicatif et combinatoire reflects this ideal quest. This study is limited to drawing attention to some of the consequences of this initiative and calling for more detailed research on the opposition lexical unit/term. It suggests the creation of appropriate techno-scientific dictionaries.
\end{abstract}

[...] quand le langage atteint le stade où ses significations sont sans ambiguïté, il peut être formalisé et exister sans aucune signification.

Henri Atlan, L'intuition du complexe et ses théorisations, 1991 : 31

\section{TERMINOLOGISATION}

Par terminologisation nous entendons la tendance à traiter - dans un but de rigueur scientifique - le vocabulaire usuel comme une vaste terminologie naturelle, accessible par observation et analyse méthodiques des productions langagières, complétée par un questionnement des locuteurs natifs. Tel semble être le cas du Dictionnaire explicatif et combinatoire (DEC).

Nous admettons que la fonction sociale première d'un dictionnaire est d'aider le locuteur en difficulté à saisir le sens et le fonctionnement des lexies posant problème (Quemada 1967: 90).

En outre, et c'est le cas du $D E C$, il peut avoir pour objet de décrire un vocabulaire scientifiquement, aussi complètement que possible, afin de servir de base de données à des dictionnaires courants ou à un modèle linguistique (en l'occurrence, au modèle transductif Sens-Texte).

\subsection{Vision topologique}

Le domaine sémantique associé au vocabulaire usuel peut être conçu a priori comme un continuum notionnel hétérogène indéfini que le lexicologue tente de discrétiser en déterminant une pseudo-partition en sous-domaines empiétants et flous de notions. Dans un dictionnaire, une acception correspond à un sous-domaine notionnel plus ou moins modelable par divers effets de sens et figures de style (Rastier 1987). 
Au contraire, le domaine terminologique serait un ensemble indéfini «dénombrable» (S. Marcus) de composants discrets, tout composant correspondant à un concept (Gentilhomme 1982a) représenté par un support langagier ou symbolique et rendu explicite moyennant une définition sine qua non - dérapages sémantiques interdits. En contexte, la correspondance paraît biunivoque : principe de monosémie (Kocourek 1982; Gouadec 1990; Gentilhomme 1984).

Ceci étant, posons la vaste question (qui, stricto sensu, dépasse les objectifs dictionnairiques) : comment un terme fonctionne-t-il dans l'esprit de ceux qui l'utilisent?

Tâchons d'apporter quelques lumières sur cette problématique qui a des impacts lexicologiques et didactiques certains.

\subsection{Hypothèse bi-systémique}

Un ensemble terminologique relève d'au moins deux systèmes. D'une part, en tant qu'unité de langue, chaque terme fonctionne comme une lexie (simple ou complexe) soumise aux contraintes linguistiques. D'autre part, le même terme s'intègre dans une discipline non linguistique, de toute autre nature. Un terme apparaît donc comme unité d'un bi-système (enchevêtrement dialectique de deux systèmes) : 1'un linguistique, vecteur de notions souples, aux frontières extensibles et poreuses, l'autre technoscientifique, exigeant des concepts rigides, à contenu strictement délimité, adapté aux raisonnements propres à ces disciplines. Ce double fonctionnement oppose fondamentalement les termes, relevant de disciplines à caractère mathématique plus ou moins accusé, aux mots «usuels» dont s'occupe le $D E C$.

\section{2. «PARADIGME EUCLIDIEN». PRÉSENTATION D'UNE TERMINOLOGIE}

a) Lors d'un exposé dogmatique linéaire ou en phase d'initiation, les termes sont présentés selon un ordre conforme à la logique de la discipline (cf. Les Éléments d'Euclide, traités, mémoires, manuels...);

b) Dans les exposés «en raccourci», les termes sont collationnés et organisés selon un mode plus pragmatique avec des éléments de preuve, et des index alphabétiques (cf. Dictionnaire raisonné des mathématiques par $\mathrm{A}$. Warusfel, fascicules Mots de l'A.P.M.E.P...);

c) Pour une consultation sporadique, au gré des besoins d'un public divers, ils figurent, sans émergence sytémique globale, dans les articles des dictionnaires dits encyclopédiques;

d) Les dictionnaires spécialisés, destinés à des publics restreints, ne recueillent que des termes et assimilés ( $c f$. Dictionnaire des Mathématiques de F. Le Lionnais, pour «l'homme cultivé du XX $\mathrm{XX}^{\mathrm{e}}$ siècle»; Dictionnaire de Mathématiques élémentaires de S. Baruk, pour apprenants et pédagogues);

e) Les termes peuvent être répartis sur des fiches techniques (La Mathématique parlée par ceux qui l'enseignent par la Commission du dictionnaire de l'A.P.M.E.P), être fixés sur des supports informatiques, etc.

Ces quelques modes de présentation n'imposent pas les mêmes contraintes, mais supposent tous un système sous-jacent de type euclidien, inexistant pour le vocabulaire usuel.

Par sa rigueur emblématique, le paradigme euclidien reste un idéal pour d'autres disciplines. Bien qu'influencé par ce paradigme, bien qu'associé à un modèle linguistique formalisé, le $D E C$ ne s'identifie à aucune des variantes ci-dessus. Au plus en retient-il certains aspects, tout en traçant sa propre voie.

Par raison de concision, le $D E C$ élabore un système de symboles avec des formules soumises à des règles explicites de bonne formation et de dérivation (notamment, pour les fonctions lexicales). Sa consultation requiert une sérieuse formation préalable. 


\section{FRONTIÈRE FLOUE}

A priori, les deux domaines - vocabulaire courant et terminologie - paraissent indépendants, le $D E C$ s'intéressant en priorité aux lexies «usuelles» et les terminologies aux termes qui, précisément, ne sont pas des lexies usuelles, générales, souples, mais qui, au contraire, sont spécifiques à chaque discipline.

La frontière s'estompe entre les deux domaines flous (Gentilhomme 1968) si l'on songe à la pénétration progressive des termes en langue (banalisation, dérapages sémantiques: Galisson 1983) avec et, surtout, à l'emploi plus ou moins spécifique des mots usuels dans les textes technoscientifiques.

En géométrie, il est courant de dire: deux figures coïncident pour n'en former qu' une seule. On voit mal un quidam soutenir, par exemple, au cours d'une visite au zoo, que deux éléphants ou deux girafes coïncident pour ne former qu' un seul éléphant ou une seule girafe (Mots: 8 ).

\section{USAGE PAR DÉLÉGATION}

Rappelons l'«usage par délégation» (Putnam 1988). Le locuteur utilise des motstermes dont il ne connaît qu'imparfaitement le contenu, en se fondant sur le fait qu'il existe des spécialistes qui les connaissent en toute rigueur - un peu comme un aveugle citant les couleurs qu'il n'a jamais perçues, mais qui sont bien connues du voyant. Il est inadmissible que, au cours d'une démonstration, un spécialiste utilise un terme sans en connaître parfaitement les tenants et les aboutissants.

L'ignorance du contenu précis des termes tels que : mécanique quantique, structure dissipative, algorithme, chaos, etc. n'empêche pas le «béotien» de les évoquer à son niveau sans proférer, ipso facto, des truismes ou des «âneries».

\subsection{Terminologie larvée}

À côté de termes considérés comme tels et dotés d'une définition explicite, il y a place pour ce que nous appelons des termes larvés (Gentilhomme 1993), non rigoureusement définis, mais véhiculant des informations spécifiques qui, dans une approche lexicographique, relèvent de la polysémie, car non réductibles à des effets de sens évidents (Mots:8).

\section{OPTIONS ET CONTRAINTES MÉTHODOLOGIQUES DU DEC}

Non-circularité. Conformément au paradigme euclidien, chaque entrée est dotée d'une définition construite à l'aide de définissants premiers, ou antérieurement définis. Les cercles vicieux sont prohibés (Marcus 1970; Warnesson 1984).

Principe d'uniformité. Les lexies porteuses de contenus comparables ( $c f$. respect, mépris. $D E C I: 21,129)$ doivent avoir une structure définitionnelle semblable... Cette règle n'est systématiquement respectée ni par la plupart des auteurs de dictionnaires, ni par les terminologues - moins «formalistes» (Sarmant 1978: ferro-, para-, diamagnétique ; Courbes mathém. 1976: ellipse, hyperbole et parabole).

\subsection{Combinatoire potentielle. Les indésirables}

La norme énoncée dans le schéma de régime admet des restrictions. Le $D E C$ cherche à prévenir la formation de syntagmes malvenus: *l'aide à la compagnie à l'administration du budget ou non souhaitables: 'la haine de Pierre des étrangers, ce que ne signalent pas les dictionnaires courants de langue.

Il existe des réserves similaires dans les textes sémiologiques hétérogènes, comprenant des unités de langue et des symboles logico-mathématiques pour raison de cohérence ou de purisme. 
Les abréviations sténographiques: ${ }^{*} X$ est + grand que $Y$ ou $*$ des points \pm alignés (par homophonie) constituent des laxismes (des barbarismes pour des censeurs chagrins), des abus de notations, «+» et «-» étant des symboles d'addition et de soustraction et non de comparaison. Tolérer la normalité de telles abréviations (sans nouvelles définitions ad hoc) revient à accepter, dans le mode sérieux, celle de un concerto pour $\bullet$ et orchestre.

$*_{\ll X}$ est $>Y »$ est mal dit, car le symbole «》» est déjà une expression prédicative, la copule est se révèle pléonastique.

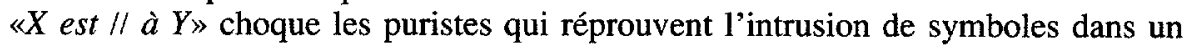
texte rédigé en langue naturelle (Lacombe 1964; Gentilhomme 1993).

Ces interdits ne sont guère mis en évidence dans les manuels et les traités, mais s'apprennent sur le tas. Leur non-signalement est parfois justifié par la croyance (le préjugé ?) qu'interdire une chose, c'est la suggérer. Il n'en reste pas moins que les écarts au «bon usage» sont cause d'embarras pour les apprenants, de soucis pour les enseignants.

Y a-t-il lieu d'en faire état dans des «dictionnaires lexico-sémiologiques»? Un dictionnaire n'a-t-il pas, peu ou prou, une vocation de mentor?

Les symboles d'unités de mesure sont souvent maltraités. Citons quelques «perlettes» pour sottisier: *La règle mesure $5 \mathrm{cms}$ (les symboles d'unités de mesure ne prennent pas la marque du pluriel par convention internationale); *Le kilog revient à $7 F 95$ (au lieu de $7,95 \mathrm{~F}$ ); *Le train roule à $70 \mathrm{~km} . \mathrm{h}$ (au lieu de $70 \mathrm{~km} / \mathrm{h}$, i.e. par heure); *5F $x 3$ pamplemousses $=15 F$ (on ne multiplie pas par des objets, mais par des nombres), etc. (Quatremer et Trotignon 1988)

Quant aux formules, un vade-mecum pédagogique devrait préciser non seulement les règles, mais aussi prévenir le néophyte contre certaines errances fréquentes, d'autant plus dangereuses qu'elles s'appuient sur une certaine réflexion réputée intelligente (Baruk 1992).

\section{HÉTÉROGÉNÉITÉ SÉMIOLOGIQUE}

Dans nombre de publications techno-scientifiques, les termes sont immergés dans des séquences hétérogènes, relevant de la sémiologie, car constituées non seulement de mots, dits usuels, et de termes spécifiques, mais aussi de symboles, de schémas, de figures, sans la présence desquels le texte devient opaque et même vide de sens. Ainsi, remplaçons, dans l'exemple ci-dessous, tous les symboles par la même lettre f.

Théorème. Pour tout couple f, il existe f, unique, tel que $f$.

Cet énoncé (Condamine 1971:15) est vide de sens pour un mathématicien. Dans une approche véri-conditionnelle, il n'est ni vrai ni faux, même approximativement et, dans une approche véri-relationnelle, il n'est comparable à aucun autre énoncé (Martin $1983: 21-22)$.

Le remplacement des formules par un symbole uniforme f équivaudrait au remplacement, dans un discours usuel, de certains mots, syntagmes ou phrases par un mot-postiche unique, par exemple schtroumpf. Une telle plaisanterie est sans doute bienvenue dans des bandes dessinées où le contexte et les images permettent le décodage; elle l'est beaucoup moins dans un corpus destiné à une analyse lexico-sémiologique. Mais il y a plus : le mot postiche des bandes dessinées admet des variantes morphologiques en fonction de son rôle syntaxique : schtroumpfer, nous schtroumpfons, schtroumpfement... possibilité que l'on n'accorde pas au symbole postiche $f$.

Toutefois, pour un linguiste, le moule langagier restant n'est pas dénué de contenu sémantique et peut encore présenter un certain intérêt. Dans un désir de compromis réaliste, ménageant les intérêts heuristiques du lexicologue-sémioticien et les possibilités matérielles d'impression, ne pourrait-on pas diversifier les postiches, à l'instar des schtroumpf? 
Quelle opinion un métalexicographe pourra-t-il se faire sur la méthodologie et la valeur du $D E C$ privé de ses symboles?

Par ailleurs, nous avons émis l'hypothèse que la coprésence des symboles et des termes n'était pas sans influence réciproque et conduisait, notamment, à repenser la notion saussurienne d'arbitrarité (Gentilhomme 1992). Comment détecter cette influence si, $a$ priori, on remplace tous les symboles et toutes les formules par un seul symbole postiche? Faire fi, par la suite, d'une telle influence relève de la «politique de l'autruche».

\section{7. «PARADIGME MENDELEÏEVIEN»}

À l'instar de la chimie, il est tentant de chercher à décomposer le contenu des lexies jusqu'à aboutir à un assortiment relativement réduit d'éléments définissants simples (primitifs). La preuve en serait d'ordre expérimental.

Ceci étant, le sens d'un mot ne se réduisant pas à un «sac» (ensemble amorphe) de sèmes, la représentation du sens se concrétise, dans le modèle Sens-Texte, par un réseau étiqueté, interprété dans le $D E C$ en un français conventionnel, compréhensible, certes, mais non sans rugosité stylistique.

N'y a-t-il pas une analogie avec la chimie organique, où des formules complexes, développées dans un espace bidimensionnel, sont linéarisées selon des conventions internationales en longues expressions alpha-numériques, linguistiquement «rugueuses»?

\section{8. «PARADIGME DYNAMIQUE ITÉRATIF»}

Il est clair que cette tentative lexicologique mendéléievienne ne manque pas d'intérêt, tant au plan théorique qu'à celui des applications aux industries de la langue. Cependant, il ne faut pas perdre de vue que ce n'est pas à partir des définitions lexicographiques qu'un «locuteur natif» acquiert l'essentiel du vocabulaire courant, mais plutôt par un processus progressif d'approximations, comparable à la résolution d'équation par itération (Gentilhomme 1982a). La consultation d'un dictionnaire n'a qu'un rôle d'appoint.

Expliquons-nous succinctement sur un exemple simple.

Soit à résoudre numériquement l'équation de forme $\mathrm{X}=\varphi(\mathrm{X})$. Le calculateur ignore la valeur exacte de $X$, mais il croit en connaître une valeur approchée $X_{0}$. Il remplace $X$ par $X_{0}$ dans le second membre et il calcule une valeur supposée améliorée: $X_{1}=\varphi\left(X_{0}\right)$. Il recommence : $X_{2}=\varphi\left(X_{1}\right)$, puis $X_{3}=\varphi\left(X_{2}\right)$ et ainsi de suite, d'où la suite $X_{0}, X_{1}, X_{2}, \ldots, X_{n}$. Si certaines conditions sont satisfaites, il se rapproche de plus en plus de la «valeur exacte».

D'une façon analogue, au départ, le locuteur ne possède qu'une idée approchée $\mathrm{X}_{0}$ du sens d'un mot $X$. Il rencontre ce mot dans des énoncés $\varphi$. Leur interprétation incite à modifier le sens prêté initalement à X. Avec ce sens amélioré $X_{1}$, il interprétera d'autres énoncés, etc. À la longue et avec un peu de chance, il se fera une idée de plus en plus juste et précise $X_{n}$ du sens du mot inconnu.

Bref, partant de connaissances réduites et vagues, à force de situer les mots dans de multiples contextes, le vocabulaire s'affine petit à petit.

Notons qu'a priori la convergence des $X_{n}$ vers une valeur limite n'est assurée, ni dans la résolution d'une équation, ni dans le processus d'acquisition du vocabulaire. Dans ce dernier cas, il y a même fort à parier que différents individus aboutiront à des contenus lexicaux (sèmes, virtuèmes, modes d'emploi connotations) tout au plus voisins, mais pas nécessairement identiques, suffisamment voisins toutefois, pour permettre la communication (Pottier 1973).

Si l'on applique cette remarque aux consultations successives des rubriques d'un dictionnaire, on se rend compte que les cercles vicieux ne sont pas aussi gênants qu'on le 
prétend, pourvu que leur diamètre ne soit pas trop petit (Marcus 1970). D'où l'intérêt de se reporter à plusieurs reprises à un dictionnaire et, notamment, de relire d'un point de vue nouveau les articles déjà consultés.

Sans doute, dans un développement terminologique linéaire, selon le paradigme euclidien, les cercles vicieux sont exclus. Le processus didactique d'acquisition, lui, n'est pas uni-linéaire et admet des boucles rétroactives. C'est souvent à la n-ième lecture d'une définition qu'on en saisit toute la portée et qu'on devient capable d'utiliser correctement le concept introduit. La comparaison algébrique ci-dessus reste valable.

\section{9. «PARADIGME HILBERTIEN»}

Selon la célèbre boutade du mathématicien allemand D. Hilbert, en substance : peu importent la nature et la dénomination des entités géométriques - plan, droite, point, ou bien table, chaise, boc de bière. Seules sont pertinentes les relations entre elles: trois bocs de bière définissent une table, deux tables peuvent avoir en commun une chaise et une seule, sur une chaise on peut placer une infinité de bocs de bière distincts, etc.

\subsection{Préformalisation. Occultation du sens}

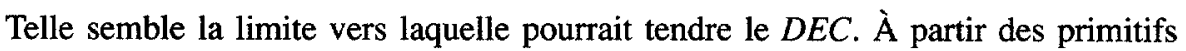
sémantiques, réductibles en théorie à des symboles abstraits, grâce aux foncteurs (prédicats, connecteurs) et aux définitions représentables par des réseaux étiquetés, le $D E C$ tente de reconstruire le lexique dans sa totalité. Selon la remarque de H. Atlan (exergue), l'élimination de toute ambiguïté conduit à la formalisation ultime, permettant un traitement automatique et, en définitive, à l'occultation méthodologique du sens humain. Il est notoire que l'ordinateur «inhumain» n'a que faire du «sens humain». Aussi, par prudence, au stade actuel du $D E C$, nous préférons l'expression préformalisation - modératrice et moins inhumaine.

\section{0. «PARADIGME GRAVITATIONNEL»}

Il peut être tentant de doter chaque domaine notionnel (\$2.2.) d'un centre de gravité représentatif (voire purement fictif), à certains égards, du domaine entier. Plus précisément, les divers «effets de sens», les significations en contexte, graviteraient, à une plus ou moins grande distance, autour d'un sens central attractif jouant un rôle de prototype sémantique (Kleiber 1990).

Il est aisé de persifler cette métaphore céleste et de citer les multiples difficultés auxquelles un modèle à structure gradientielle reste impuissant à faire face. Notons que l'image solaire n'est qu'une réplique pittoresque des arborescences structurant les rubriques dictionnairiques.

Grosso modo, dans la perspective componentielle, un contenu est caractérisé par un assortiment de sèmes et de virtuèmes. Les dérapages sémantiques résulteraient de la dissimilation ou de l'activation de certains traits créant des sous-domaines parasynonymiques plus ou moins attachés au centre de gravité (Martin 1976; Rastier 1987).

Le $D E C$ regroupe les réseaux parasynonymiques. Chaque réseau est justifié par l'existence d'une lexie et a droit à une entrée particulière. Le lien attractif est réalisé par l'existence d'intersections non triviales.

Une fois le «soleil» pressenti, force est de chercher à mettre en place des planètes, des astéroïdes, voire des comètes sémantiques.

\section{1. «PARADIGME DISSIPATIF»}

Dans son essence, le paradigme itératif est clos. Le sens se construit au cours d'un processus répétitif, à l'intérieur d'un système doté implicitement d'une frontière. Dans 
toute démarche vécue par les individus réels, deux autres facteurs sont à considérer. D'une part, la rencontre de telle ou telle occurrence, de tel ou tel contexte ne relève pas d'une loi prédéterminée, ni même d'une démarche préconçue, mais constitue, pour une large part, un événement aléatoire qui déséquilibre (peut-être de façon minime, mais non négligeable à la longue) le système en développement et amorce son devenir à l'intérieur d'un certain angle de contingence, selon une fourchette liée au monde des possibles et à l'univers des croyances (Martin 1983).

D'autre part, on ne peut faire abstraction des connaissances encyclopédiques et des expériences vécues par l'individu qui enrichissent le contenu cognitif des lexies en cause et de leurs interrelations. Cet apport cognitif (non shannonien) étranger au système lexical stricto sensu, joue un rôle moteur; métaphoriquement, il insuffle «une énergie externe» «dissipée» au cours de l'élaboration et de l'appropriation du sens. Le trajet cognitif ne peut être parcouru en sens inverse. La «flèche du temps» est irréversible. L'apport dissipatif semble lié aux phénomènes comme la critique d'une définition donnée, la compréhension de ce que subsume une définition abstraite, la découverte d'un sens pour une expression conçue précisément pour ne pas en avoir (Grize 1990: hypothèse de générosité).

\section{TRANSFERT DE MÉTHODOLOGIES}

Il y aurait beaucoup à dire sur le transfert des méthodologies lexicologiques aux terminologies et vice-versa. Nous nous limiterons à quelques remarques concernant le $D E C$.

Le grand mérite du $D E C$ est d'avoir affiné, développé, voire rénové, dans une optique déterminée, certaines méthodes lexicales traditionnelles. Examinons celles qui nous paraissent susceptibles d'être appliquées en terminologie, à savoir la mise en évidence des «fonctions lexicales» et des «participants (actants) sémantiques».

\subsection{Fonctions lexicales (FL)}

L'objet des FL standard (DEC 1\&3) est de recenser et de décrire de façon condensée une catégorie d'expressions figées et semi-figées usuelles. Par analogie avec les fonctions mathématiques, les FL associent, sous certaines conditions, à une lexie clé, ou argument, une liste d'expressions idiomatiques ou valeurs. La plupart des FL sont porteuses d'un sens plus ou moins abstrait, comme Magn - l'intensité, Degrad - la perte des qualités pertinentes, Ver - la satisfaction de la vocation fondamentale, Bon - une qualification laudative vague, Sing - la désignation d'une unité minimale comptable, Mult - celle d'un ensemble normalement constitué, Anti - un antonyme, Gener - un mot générique dont la combinaison avec le mot clé est synonyme du mot lui-même, etc.

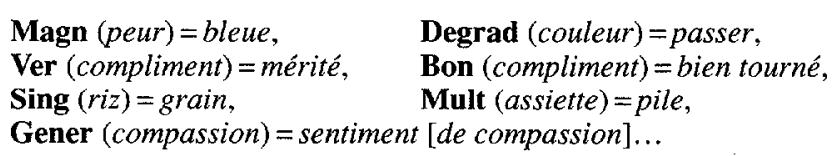

Les FL sont susceptibles de se combiner entre elles:

AntiMagnSon $($ parler $)=$ chuchoter, parler à voix basse...

Dans le domaine des sciences et des techniques, la pertinence variant avec les disciplines en cause, certaines fonctions lexicales méritent d'être exploitées, d'autres paraissent sans intérêt. En outre l'assortiment des valeurs d'une FL associées à un argument donné est tributaire de la situation d'énonciation. Les valeurs «trop fleuries» ne font pas 
«sérieux» dans une prose académique et sont éliminées. Ainsi, en météorologie (par exemple à la radio) on n'a guère de chance de rencontrer:

Magn $($ vent $)=$ à décorner les boufs, $($ pleuvoir $)=$ des cordes, comme vache qui pisse.

De même que pour le langage courant, les valeurs proposées ne sont pas toutes absolument impératives. Elles restent, cependant, contrôlées par le consensus d'une norme qui s'est instaurée dans le parler courant des spécialistes. Ainsi, pour des contextes appropriés, on peut proposer:

Magn (tension) $=$ haute (en électricite), AntiMagn $(-)=$ basse;
AdvMagn (tension) = sous haute, AdvAntiMagn $(-)=$ sous basse;

Magn $($ pression $)=$ forte, $\quad$ AntiMagn $(-)=$ faible ;

Magn (probabilitê $)=$ voisine de un, AntiMagn $(-)=$ voisine de zéro,

Magn (chauffer) $=$ au rouge cerise $<$ à blanc,

Degrad (hyperbole) = dégénérer [se réduire à deux droites concourantes, dans l'équation :

$a x y+b x+c y+d+0$, quand $d$ tend vers zéro];

Degrad (batterie) $=$ se sulfater, AntiDegrad $(-)=$ se régénérer ;

Degrad $($ granite $)=$ pourrir,$($ roche $)=s^{\prime}$ effriter,$($ grès $)=$ se désagréger

Ver (solution [math.]) = exacte, AntiVer $(-)=$ fausse.

Bien entendu, on observe des niveaux de langage spécifiques:

Bon (solution [math. «familier»]) =élégante, AntiBon $(-)=$ lourde

Bon (fonction [math. «familier»]) = bonne, i.e. qui ne présente pas de singularités exigeant une approche plus délicate, l'utilisation de théories sophistiquées.

Sing (ensemble) $=$ élément,

Mult $($ droites $)=$ faisceau,$(\rightarrow$ bactéries $=$ colonie..

Certaines FL standard n'intéressent pas toutes les disciplines.

La FL Son, qui indique le verbe associé à un son ou à un cri spécifique, peut intervenir en ornithologie, en acoustique... rarement dans de nombreuses autres disciplines, jamais en mathématiques.

Son $($ caille $)=$ courcailler,$($ huppe $)=$ pupuler,$($ milan $)=$ huir,$($ coq de bruyère $)=$ dodeldir

Son (tuyau sonore, corde vibrante) = résonner,

Son $($ phonolite $)=$ résonne, sonner [roche volcanique sonore sous le choc], etc.

En marge des FL standard, il est possible de concevoir des FL non standard, concernant plus particulièrement une discipline donnée.

Ainsi, en zoologie, on pourrait envisager une FL pour le terme désignant l'animal du sexe opposé, ou encore le petit d'un animal :
Sex $($ cheval $)=$ jument,
$($ vache $)=$ taureau,
Infan $($ chat $)=$ chaton,
$($ poule $)=$ poussin,
$($ loup $)=$ louve, etc.
$($ loup $)=$ louveteau, etc.

En géographie, en économie, les noms des monnaies de divers pays peuvent être présentés comme des valeurs de FL ad hoc:

Monn $($ Italie $)=$ lire,$($ Russie $)=$ rouble,$($ Sri Lanka $)=$ roupie, etc.

Certes, la nature de la monnaie relève des connaissances dites encyclopédiques, cependant, la façon de la nommer appartient au lexique.

Les services comptabilité (et les interprètes internationaux) doivent connaître le nom de la somme d'argent perçue par certaines personnes, dans diverses conditions :

Sal $($ ouvrier $)=$ salaire,$($ fonctionnaire $)=$ traitement,$($ parlementaire $)=$ indémnité,$($ chômeur $)$ $=$ allocation, $($ avocat $)=$ provision, $($ notaire $)=$ vacation, honoraire, (médecin $)=$ honoraire, etc. 
Le champ d'exploration des FL est immense; nous nous contentons ici d'en suggérer l'étude.

\subsection{Participants et actants sémantiques}

Nous relevons dans le $D E C$ III : 156 :

Cours de $\mathrm{X}$ sur $\mathrm{Y}$ de $\mathrm{Z}[\grave{a} \mathrm{~W}] \grave{a} \mathrm{U}=$ Portion d'enseignement organisé et constituant un tout portant sur $\mathrm{Y}[\ldots]$ donné dans un établissement $\mathrm{U}$ d'enseignement secondaire ou supérieur par X à W sur Y au sein d'une matière $Z$.

En effet, pris dans cette acception, le lexème cours fait intervenir explicitement ou implicitement un certain nombre de participants sémantiques : l'enseignant X, l'apprenant W, le sujet abordé $Y$ dans la matière en cause $Z$, à l'intérieur d'un établisement scolaire U.

Les participants sémantiques pourront être effectivement introduits dans un texte, compte tenu des moyens syntaxo-morphologiques prévus par la langue, et deviennent des actants au sens de Tesnière (1959). Exemple:

Cours dispensés à la Sorbonne $U$ (de mathématiques supérieures $Z$ ) sur la résolution des équations aux dérivées partielles $Y$, aux étudiants de deuxième année $W$, assurés par un nouvel assistant $X$.

Les participants sémantiques et les moyens relèvent de la description du lexème considéré, leur connaissance étant nécessaire pour pouvoir l'utiliser en discours.

Le même problème se pose pour les termes, certains circonstants devenant, dans le bi-système, des actants à part entière (Gentilhomme 1982b) :

Division de $X$ par $Y$ avec un reste $R$,

Cristallisation de $X$, dans $L$, sous la forme $F$, à la température $T$, à la pression $P$,

Arrangement de $M$ objets, $P$ à $P$, avec (sans) répétition,...

Les actants sémantiques portent parfois des noms génériques spécifiques que désignent des FL appropriées : $\mathbf{S}_{\mathbf{1}}, \mathbf{S}_{\mathbf{2}}, \mathbf{S}_{\mathbf{3}}, \ldots, \mathbf{S}_{\mathbf{n}}$

$\mathbf{S}_{\mathbf{1}}($ division $)=$ diviseur, $\mathbf{S}_{\mathbf{2}}(-)=$ dividende, $\mathbf{S}_{\mathbf{3}}(-)=$ quotient, $\mathbf{S}_{\mathbf{4}}(-)=$ reste

$\mathbf{S}_{\mathbf{1}}$ (solution) = solvant, $\mathbf{S}_{\mathbf{2}}(-)=$ soluté, $\mathbf{S}_{\mathbf{3}}(-)=$ concentration,

$c f$. solution $\left(\mathbf{S}_{1}\right)$ aqueuse $\left(\mathbf{S}_{3}\right)$ binormale 2 moles par litre] $\left(\mathbf{S}_{2}\right)$ de soude.

La FL $\mathbf{A b l e}_{\mathbf{n}}$ fournit l'adjectif de possibilité, l'indice précise l'actant concerné :

Able $_{2}$ (division) $=$ divisible, $\left[\left(\mathbf{S}_{1}\right) 7\right.$ «peut diviser $\gg\left(\mathbf{S}_{2}\right)$ 35],

Able $_{2}$ (intégrer $)=$ intégrable $\left[\left(\mathbf{S}_{2}\right)\right.$ la fonction $f(x)=x e^{x}$ est intégrable $\left[\left(\mathbf{S}_{\mathbf{m o d}}\right)\right.$ par parties $]$,

$\mathbf{A b l e}_{2}($ solution $)=$ soluble, En effet, $\mathbf{A b l e}_{1}(\rightarrow$ serait : susceptible de dissoudre, être un solvant,

l'adjectif correspondant manque apparemment.

Rappelons que J.-L. Descamps et coll. (1976 CREDIF et 1992) notent la présence, dans le contexte large d'une occurrence-lexie donnée, de certains éléments d'information spécifiques qui, dans une prospection lexicologique plus profonde; peuvent jouer le rôle de participants sémantiques.

Le concept de fonction appelle pour le moins ceux d'argument, de valeur et de domaine de définition qui peuvent être éparpillés dans le texte.

Par ailleurs, l'introduction en discours de certains noms requiert l'emploi de verbes spécifiques, à peu près vide de sens (dans le contexte), comme c'est le cas de facteur et acide, ci-dessous : 
Mettre $\left(\mathbf{S}_{2}\right)$ qqchose en facteur (= factoriser),

$\left(\mathbf{S}_{\mathbf{1}}\right)$ un acide attaque $\left(\mathbf{S}_{2}\right)$ un métal, où attaquer n'évoque pas la métaphore du combat, mais suggère la réalisation d'un processus propre à un acide.

On note: Oper $_{1}($ facteur $)=$ mettre $\left[\right.$ en], Real $_{1}($ acide $)=$ attaquer

\section{SYNONYMIE, POLYSÉMIE}

Le problème de la synonymie exacte et approchée est central (tant en linguistique générale qu'en terminologie). Il est hors de question ici de l'aborder en profondeur. Nous nous limiterons à rappeler qu'il se pose de façon particulière dans la littérature technoscientifique.

Dans le modèle transductif Sens-Texte, l'identité de sens (exacte ou approchée) est reconnue de prime abord comme un fait d'expérience. Deux expressions (mots, phrasèmes, phrases) sont synonymes si un nombre suffisant de locuteurs les considèrent comme tels. Le sens est alors défini comme un invariant commun.

Au niveau logique, la synonymie de deux expressions implique des définitions équivalentes, l'implication réciproque et, par suite, la cosubstituabilité, aux rugosités de style près. En particulier, une lexie est substituable à sa définition et vice versa.

\subsection{Sens, référent, information}

Il importe de distinguer identité de sens, d'identité de référent et identité d'information. Certes, l'identité de sens paraît entraîner celle du référent et celle de l'information. La réciproque est manifestement fausse.

$\mathrm{Si}$, pour établir l'identité d'information véhiculée par deux expressions, on doit faire appel à des connaissances extralinguistiques, logiques ou mathématiques, leurs sens lexicaux ne sont pas identiques (DEC III, pp. $11 \& 54)$.

Que faut-il entendre par référent en mathématiques?

Par exemple, pour un cercle, faut-il considérer la classe de tous les cercles existants à l'heure actuelle, qui ont existé et qui vont exister? Ce n'est là qu'une vue de l'esprit et, d'ailleurs, un géomètre ne saurait raisonner avec un pseudo-concept de cette sorte. L'inadéquation d'une telle approche est encore plus patente si, au lieu d'une figure géométrique, on envisage des entités comme les nombres transfinis de Cantor ou des opérateurs comme la divergence (d'un vecteur), le laplacien, etc. qui ne sont préhensibles que moyennant une haute culture mathématique.

Doit-on envisager une représentation commune qui se construit dans le cerveau des individus à la vue d'un assortiment de «cercles concrets»? Une telle représentation s'apparente plutôt à une notion et non à un concept, notion formalisable par le concept de classe objet (selon la terminologie de l'École de Neuchâtel). Une classe objet, par opposition à la classe distributive, est susceptible de s'enrichir sans cesse d'ingrédients nouveaux, qui ne sont pas obligatoirement des sous-classes ou des éléments. Quoi qu'il en soit, comme ci-dessus, une telle notion ne permet pas un raisonnement mathématique rigoureux; néanmoins, elle peut jouer un rôle important pour l'intuition créative et pour la néologie.

Notons que les réserves relatives à la classe-objet n'interdisent pas toute forme de raisonnement. Au contraire, dans la vie courante, la plupart des individus ne prennent en compte que des notions, et non des concepts. Leurs déductions peuvent être parfaitement opérantes. Ce faisant, ils suivent une logique que J.-B. Grize appelle «naturelle», différente de la logique formelle des mathématiques.

À l'instar des sciences dures, la terminologisation cherche à associer à chaque lexie un concept, rendu explicite grâce à une définition rigoureuse et qui, dans l'optique du 
paradigme gravitationnel mentionné ci-dessus, pourrait jouer un rôle de centre de gravité d'un domaine notionnel.

\subsection{Synonymies approximatives}

Le problème de proximité (distance) est incontournable. Selon la nature de la proximité, le $D E C$ note la synonymie à l'aide des FL Syn (synonyme exact), Syn $\subset$ (plus étroit), $\mathbf{S y n} \mathbf{n}_{\supset}$ (plus large) et $\mathbf{S y n} \mathbf{n}_{\cap}$ (coïncidence partielle)

$$
\begin{array}{ll}
\text { Syn }(\text { mémoire })=\text { souvenir }, & \mathbf{S y n}_{\subset}(\text { colère })=\text { rage }, \\
\mathbf{S y n}_{\supset} \text { (rétorquer) }=\text { objecter }, & \left.\mathbf{S y n}_{\cap} \text { (joie }\right)=\text { plaisir } .
\end{array}
$$

Il va de soi que la mise en pratique des principes généraux ne va pas sans difficultés. Si l'on en croit les spécialistes, il serait légitime d'écrire:

Syn (être un élément de) = appartenir à [en parlant d'ensemble]

Syn (hypocycloïde de Steiner) = Deltoïde [noms différents pour une même courbe]

Syn $($ gaz carbonique $)=$ dioxyde de carbone $\left[\right.$ même gaz $\left.\mathrm{CO}_{2}\right]$

et, entre symboles:

Syn $(<$ [est plus petit que]) $=\geq$ [n'est pas plus grand que ou égal à]

Syn $\left(f^{\prime}(x)\right)=d f(x) / d x$ [dérivée de la fonction $f$ par rapport à $\mathrm{x}$ ]

La «synonymie exacte» est justifiée par l'identité d'information perçue, par celle du référent-définition et par la cosubstitution tolérable dans, apparemment, la plupart des contextes courants.

Pour préciser la nature de la synonymie, le $D E C$ fait appel aux définitions qu'il propose. Ainsi, le synonyme est considéré plus large si et seulement si sa définition est incluse dans celle du synonyme plus étroit.

Cependant, on peut se demander dans quelle mesure les notions de synonymie plus large et plus étroite s'apparentent à celles d'hyperonymie et d'hyponymie, telles qu'on les rencontre en linguistique. Si tel n'est pas le cas, comment faire la distinction, selon quels critères? Les notations:

$\mathbf{S y n}_{\subset}($ cercle $)=$ circonférence, $\quad \mathbf{S y n}_{\cap}($ courbe $)=$ ligne, $\quad \mathbf{S y n}_{\supset}($ fraction $)=$ nombre,

$\mathbf{S y n}_{\subset}$ (équivalence) $=$ égalité, $\quad \mathbf{S y n}_{\supset}($ égalité $)=$ identité [remarquable en alg. élémentaire].

$\mathbf{S y n}_{\cap}($ appartient $\hat{a})=$ est inclus dans

décrivent-elles une réalité lexicale pertinente? Sans parler d'exemples comme:

$\mathbf{S y n}_{\supset}($ anneaux de Borromée $)=($ Noud emboité $)$, [cf. la théorie des nouds],

$\mathbf{S y n}_{\cap}($ Horicycle $)=($ Endocycle $)$, [en Pangéométrie de Lobatchevski],

dont le contenu n'est accessible qu'à des spécialistes qui, eux, n'ont cure de ces considérations. Nous posons le problème.

\subsection{Pléonasme consacré}

Une FL assez curieuse Gener indique un mot générique dont la combinaison syntaxique avec le mot clé est synonyme du mot clé lui-même comme : sentiment dans sentiment de colère. On pourrait de même écrire :

Gener (addition, soustraction, multiplication, division, élévation à une puissance...) = opération,

([un] entier, rationnel) $=$ nombre,

(cristallisation, lyophilisation, auto-oxydation-réduction) $=$ processus,

(butane, chlore, méthylène, dioxyde d'azote) $=$ gaz, 
(agaricacées, ombellifères, félidés) = famille,

(convexe, concave, rectiligne $)=$ de forme,

puisque parler de l'opération d'addition, du processus de cristallisation, du gaz butane, de la famille des agaricacées, d'être de forme convexe n'est rien d'autre que de parler simplement de l'addition, de cristallisation, du butane, des agaricacées ou d'être convexe.

Il convient de remarquer que ces mêmes termes, redondants en l'occurrence, peuvent, avec d'autres mots clés ou avec certains contextes, être porteurs d'information utile. Ainsi, gaz ammoniac, ou gaz acide chlohydrique s'opposent aux solutions de ces mêmes gaz qu'on manie plus commodément sous forme de solution liquide.

On peut même, le cas échéant, opposer le phénomène général à un processus particulier.

D'une façon générale, il se trouve qu'avec le même mot clé, dans certains contextes l'indication du genre prochain constitue une redondance lexicale manifeste, mais, dans d'autres, elle est tolérée, voire bienvenue, notamment lorsque, avec propos, elle lève une ambiguïté. Tel peut être le cas de termes polysémiques comme permutation, arrangement, combinaison qui, en analyse combinatoire, sont susceptibles de désigner soit une opération, l'application d'un ensemble sur un autre, soit la séquence, résultat de cette opération.

Il est clair que, dans l'enseignement, il importe de faire des rappels, d'insister sur des faits bien connus. Un débutant peut fort bien confondre une famille avec une espèce, un ordre ou une quelconque autre classe, sous- ou sur-classe taxinomique.

La question de redondance peut être soulevée et discutée également au niveau métalinguistique, notamment au sujet de l'emploi autonyme. Dans «L'expression: lieu géométrique a été remplacée avantageusement par le terme ensemble», est-il vraiment nécessaire de spécifier qu'il s'agit d'une expression et d'un terme?

\subsection{Pataquès définitionnels}

Un définissant possède, en général, plusieurs acceptions que le $D E C$ identifie par des indices pour guider le bon choix. Une telle façon de procéder est conforme au souci majeur du terminologue : éviter toute dyscompréhension.

Ainsi, en tant que terme mathématique, le mot axe, sans autre spécification, est hautement polysémique. Le dictionnaire de Le Lionnais en propose 25 acceptions.

Or, par nature, un terme est ambivalent. Il joue d'une part un rôle de lexie et admet à ce titre une définition lexicologique qui, en discours, n'interdit pas les effets de sens (Rastier 1983); mais, par ailleurs, il possède, à l'intérieur de la discipline considérée, une définition sine qua non, laquelle exclut tout dérapage sémantique (Gentilhomme 1982a et 1992).

En langue usuelle, parler de probabilité d'un événement c'est faire entendre, plus ou moins, d'une part, que celui-ci n'est pas impossible, d'autre part qu'il n'est pas certain, enfin qu'il a certaines chances de se produire. L'événement probable se situe bien dans le monde des possibles, mais on ne peut assurer, sans réserves, qu'il se trouve sur le rameau des attentes (Martin 1983).

Le mot probabilité exprime une notion intuitive évasive, non évaluable selon une technique objective, les limites entre ces trois valeurs ne sont pas rigoureusement tracées. D'où: $\mathbf{S y n}_{\cap}($ probable $)=$ non impossible, vraisemblable, plausible, prévisible, incertain, contingent...

En mathématiques d'antan (mais présentes au stade d'initiation), la probabilité d'un événement apparaît comme un nombre déterminé, compris entre 0 et 1 . Ce nombre est obtenu, dans une expérience donnée, comme le quotient du nombre de cas favorables au nombre de cas possibles ou, encore, dans une approche statisticienne, c'est la limite (si elle existe) de la fréquence lorsque le nombre d'expériences augmente indéfiniment (Borel 1909 et 1946; Frechet 1955; Encyclopédia Universalis 1989, t.18:1015 sqq.).

En Mathématiques de notre temps, la définition est élargie et, en même temps, vidée de tout contenu expérimental et psychologique: «La probabilité d'un événement $\mathrm{A}$ d'un 
espace probabilisé ( $\Omega, A, \mathrm{P})$ est le réel $\mathrm{P}(\mathrm{A})$.» Elle cesse d'être accessible à un non-initié. Il n'en ressent pas spontanément la filiation avec les concepts dégagés jadis par Fermat et Pascal.

Quelque part, dans son mémoire, le mathématicien fera comprendre au lecteur quelle est, en l'occurrence, la définition adoptée.

En revanche, le terme paramathématique plausibilité est proposé précisément aux cas où la probabilité ne peut être calculée, car elle cesse d'avoir un sens pour des raisons théoriques opérationnelles. Il en est ainsi de la conjecture de Golbach qui, néanmoins, rentre dans le monde des attentes, mais pour des raisons expérimentales objectivables (PÒLYA 1954).

À la probabilité mathématique, s'oppose la probabilité subjective souvent fleurie d'un nombre, qui symbolise une impression personnelle, ne résultant ni d'une statistique contrôlée, ni d'un quelconque calcul théorique. Si la liaison par le symbole $\mathbf{S y n}_{\cap}$ reste valable lorsque les mots probabilité et plausibilité sont utilisés avec leur fonction de lexies (même à l'intérieur d'un texte spécialisé), elle perd sa pertinence quand ces mots deviennent des termes en contexte démonstratif. Le lexicographe, chargé de la rédaction de l'article Probabilité dans un dictionnaire encyclopédique, examinera les diverses acceptions, usuelles, puis techniques, les unes à la suite des autres, tout en essayant de trouver des paraphrases accessibles au profane.

Dans un contexte littéraire, l'univocité rigide du sens n'est pas toujours impérative. Une certaine indétermination peut même être souhaitable. Pour qu'un texte soit riche, il n'est pas mauvais qu'il puisse être saisi de diverses façons : chaque lecteur y trouve son bien.

\section{ISOCHORISME LEXICAL}

Pour mieux faire saisir notre problématique, présentons-la d'abord sous une forme plaisante. Soit le texte partiellement orthographié:

Dans la bonne ville de $[\mathbf{s o}]_{1}$, un $\left[\mathbf{s o}_{2}\right.$ transportait un $[\mathbf{s o}]_{3}$ dans un $[\mathbf{s o}]_{4}$, le $[\mathbf{s o}]_{5}$ fit un méchant $[\mathbf{s o}]_{6}$ et les trois $[\mathbf{s o}]_{7}$ tombèrent par terre.

Quatre hétérographes (formes fléchies non considérées) correspondent à la transcription phonétique [so]. Le contexte et certaines connaissances encyclopédiques permettent plus ou moins de les identifier:

$[\text { [so] }]_{1}$ : Sceaux [la bonne ville de...]; [so $]_{2}$ : sot [qq'un transportait qq. chose];

[so] $]_{3}$ : sceau, seau ou sot [petite hésitation, le choix se fixe habituellement sur sceau];

$[\text { so }]_{4}$ : seau [dans un...]; $\quad\left[\right.$ so $_{5}$ : sot [fit qq. chose] ;

$[\text { so }]_{6}:$ saut,$[$ Oper $($ saut $)=$ faire et contexte $] ; \quad\left[\right.$ so $_{7}:$ impossible à orthographier.

Proposons la solution conforme à l'univers de croyance de la plupart des locuteurs :

Dans la bonne ville de Sceaux, un sot transportait un sceau dans un seau, le sot fit un méchant saut et les trois [so] tombèrent par terre.

\subsection{Hypothèse paradoxale}

Dans la chaîne écrite, à l'emplacement de [so $]_{7}$, se trouvent non pas une lexie, mais trois lexies orthographiées: sot, sceau et seau, ce qui n'est pas le cas des emplacements, de saut et Sceaux. Si l'on peut parler de polysémie résoluble pour les cinq premiers [so], il est essentiel, pour conserver tout son sel à la plaisanterie, d'avoir présents à l'esprit, simultanément, trois signifiés distincts pour un seul signifiant, d'où le terme d'isochorisme emprunté à la Textique (J. Ricardou).

Notre hypothèse est que tel est également le cas des termes technoscientifiques. Précisons notre propos.

Selon la nature de sa démarche cognitive (activité discursive ou logique), le scientifique se réfère au signifié conceptuel ou au signifié notionnel (tous les deux présents 
dans une occurrence textuelle) et c'est la dualité du statut sémantique qui opposerait la lexie monostatutaire au terme amphibie (Gentilhomme 1992; 1993).

La compréhension en profondeur d'un discours technoscientifque ne se réduit pas à suivre pas à pas chaque chaînon logique conforme aux contenus conceptuels des unités constitutives, bien qu'une telle préhension soit indispensable. Dans un parcours plus large, stimulé par les contenus notionnels des constituants, grâce à une perception contrapuntique globale, il y a émergence d'une unité discursive originale, porteuse de l'information complète (Gentilhomme 1988).

\section{CONCLUSION}

La terminologisation consiste à vouloir traiter (du moins en apparence) les notions comme des concepts, donc les lexies comme des termes multisémiques. Dans un souci de rigueur scientifique, le $D E C$ s'inspire des paradigmes euclidien, mendéleïevien et même hilbertien. Une telle façon de faire est inévitable si l'on envisage les applications informatisées, ne serait-ce qu'en vue des systèmes experts. Cependant, si l'on souhaite saisir de plus près le fonctionnement du lexique en langue, et tenir compte de la vocation didactique d'un dictionnaire, force est de tirer parti des paradigmes gravitationnel, itératif et dissipatif.

Pour ce qui est des termes, il convient de porter son attention sur leur nature hybride suite à leur double fonctionnement à l'intérieur d'un bi-système linguisticotechnologique.

Certaines méthodes de description lexicales méritent d'être adaptées aux dictionnaires terminologiques, entre autres: le relevé des participants sémantiques avec leur mode d'expression et des valeurs usitées des fonctions lexicales utiles.

Est-il raisonnable de rêver à des dictionnaires terminologiques qui prennent en compte le double statut des termes?

\section{RÉFÉRENCES}

BARUK, S. (1992) : Dictionnaire de mathématiques élémentaires, Paris, Seuil.

BOREL, E. (1909) : Éléments de la théorie des probabilités, Paris, Hermann, $3^{e}$ éd. 1924.

BOREL, E. (1946) : Les mathématiques et la vie, Paris, PUF, «Que sais-je?».

Bulletin de l'A.P.M.E.P. (Association des Professeurs de l'Enseignement Public).

CANDEL, D. (1979) : «La présentation par domaines des emplois scientifiques et techniques dans quelques dictionnaires de langue», $L . F ., 43$, pp. 100-115.

CANDEL, D. (1983) : «Réflexions sur l'utilisation de textes scientifiques dans un dictionnaire de langue», É.L.A., 51, pp. 21-33.

CANDEL, D. (1984) : «Une approche de la langue des physiciens», L.F., 64, pp. 93-107.

CONDAMINE, M. (1971): Algèbre, terminales C-D-E, Paris, Delagrave.

Courbes mathématiques (1976) : Revue du Palais de la découverte, $\mathrm{n}^{\circ}$ spécial 8, Paris.

CREDIF (1976): Dictionnaire contextuel de français pour la géologie : Essai de classement d' une concordance de français scientifique et étude critique, rédigé par: J.-L. Descamps, G. Gagnon, M. T. Gautier, D. Lehman, ..., Paris, Didier, 2 vol.

DAGOGNET, F. (1969): Tableaux et langages de la chimie, Paris, éd. du Seuil.

DEC (Dictionnaire explicatif et combinatoire du français contemporain. Recherches lexico-sémantiques), T. I, 1984, II, 1988, et III, 1992, par I. A. Mel'cuk et coll. [N. Arbatchewsky-Jumarie, L. Dagenais, L. Elnitsky, L. Iordanskaja, A. Lessard, S. Mantha, M.-N. Lefebvre...], Montréal, Les Presses de l'Université de Montréal. DESCAMPS, J.-L. et coll. (1992) : Sémantique \& concordances, coll. «Saint-Cloud», Paris, Klincksieck. Encyclopedia Universalis (éd. 1989) : France S.A., Paris.

FRECHET, M. (1955) : Les mathématiques et le concret, Paris, PUF, 438 p.

GALISSON, Robert (1983): Des mots pour communiquer. Éléments de lexicométhodologie, Paris, CLE international.

GENTILHOMME, Y. (1968) : «Les ensembles flous en linguistique», Cahiers de linguistique théorique et appliquée, 5, Bucarest, pp. 47-65. 
GENTILHOMME, Y. (1982a): «De la notion de notion à la notion de concept. Processus dynamique itératif d'acquisition des notions. Conséquences lexicales et didactiques», T.C.R.S. (Travaux du Centre de Recherches Sémiologiques), 42, Université de Neuchâtel, pp. 66-89.

GENTILHOMME, Y. (1982b) : «Lecture d'un texte scientifique», Pratiques, pp. 100-115.

GENTILHOMME, Y. (1988) : «Compréhension en surface et compréhension en profondeur d'un texte technoscientifique. Impact sur la traduction et sur l'enseignement-apprentissage d'une langue étrangère», La pensée scientifique et ses discours, Besançon, Actes du Colloque ANEFLE-CLAB, pp. 51-110.

GENTILHOMME, Y. (1984): «Les faces cachées du discours scientifique», L.F., 64, pp. 29-37.

GENTILHOMME, Y. (1990) : «Les lubrifiants didactiques», Recherches en linguistique étrangère, XIV, Annales littéraires de l'Université de Besançon, diff. Paris, «Les Belles Lettres», pp. 25-91.

GENTILHOMME, Y. (1992) : «L'éclatement du signifé dans les discours techno-scientifiques», Signifiant, référent, réel, CsRLE, vol.20, Annales littéraires de l'Université de Besançon, diff. Paris, «Les Belles Lettres», pp. 29-66.

GENTILHOMME, Y. (1993) : «Reflexions sur les discours scientifiques», Fachsprachentheorie BdI Fachsprachliche Terminologie, Begriffs- und Sachsysteme. Methodologie, betreut und herausgegeben von Th. Bungarten, Attikon Verlag, Tostedt, pp. 430-494.

GOUADEC, D. (1990) : Terminologie, AFNOR Gestion.

GRIZE, J.-B. (1990) : Logique et langage, Gap, OPHRYS.

JAKOBSON, R. (1963) : Essai de linguistique générale, t.1, trad. de l'anglais par Nicolas Ruwet, Paris, éd. de Minuit, coll. «points».

KLEIBER, G. (1990): La sémantique du prototype, catégorie et sens lexical, Paris, PUF.

KOCOUREK, R. (1982) : La langue française de la technique et de la science, Wiesbaden, Oscar Brandstetter Verlag GMBH \& Co, K.G.

La Commission du dictionnaire de l'A.P.M.E.P. (1967-1993) : «La mathématique par ceux qui l'enseignent», Fiches publiées périodiquement, après discussion et publications critiques dans le Bulletin de L'A.P.M.E.P.

LACOMBE, D. (1964) : «Sur les mots et les symboles», Bulletin de l'A.P.M.E.P., 239, pp. 343-355.

LE LIONNAIS, F. (1979): Dictionnaire des Mathématiques, Alain Bouvier et Michel George (dir.), Paris, PUF.

LOMBARDI, H. (1989) : «Mathematiques constructives : hier et aujourd'hui», Bulletin de l'irem de Besançon, mathématiques vivantes, 40, Univ. de Franche-Comté, pp. 3-18.

MARCUS, S. (1970) : «Définitions logiques et définitions lexicographiques», Langages, 19, pp. 87-91.

MARTIN, R. (1976) : Inférence, antonymie, et paraphrase. Eléments pour une théorie sémantique, Paris, Klincksieck, 176 p.

MARTIN, R. (1983) : Pour une logique du sens, Paris PUF.

MOTS Réflexions sur quelques mots-clés à l'usage des instituteurs et des professeurs, Ont paru, depuis 1974, neuf fascicules. Fasc. 8 , : Mots clés, mots flous.

PÒLYA, G. (1954) : Les mathématiques et le raisonnement "plausible», trad. fr. 1958, Gauthier-Villars.

POTTIER, B. (dir.) (1973) : Le langage, coll. «Les dictionnaires du savoir moderne», Paris, éd. Retz (Centre d'Étude et de Promotion de la Lecture).

PUTNAM, H. (1988) : Représentation el réalité, trad. fr. 1990, Paris, Gallimard.

QUATREMER, R. et J.-P. TROTIGNON (1988): Précis. Unités et grandeurs, système international (SI), symboles et normalisation, AFNOR, $3^{\mathrm{e}}$ éd., Paris, Nathan.

QUEMADA, B. (1967) : Les dictionnaires du français moderne, 1539-1863, Paris, Didier.

QUEMADA, B. (1990): Dictionnaire et Lexicographie, $\mathrm{n}^{\circ} 1$, «Autour d'un dictionnaire, Le Trésor de la Langue française, témoignages d'atelier et voies nouvelles», CNRS, Paris, Didier Érudition.

RASTIER, F. (1987) : Sémantique interprétative, Paris, PUF.

SARMANT, J.-P. (1978) : Dictionnaire de physique, Paris, Hachette, 1850 articles.

TESNIÈRE, L. (1959) : Éléments de syntaxe structurale, Paris, Klincksieck.

WARNESSON-QUEMADA, I. (1984) : Lexicographie et ordinateur. Recherches sur les applications de l'informatique aux élaborations lexi...cographiques, Thèse de $3^{\mathrm{e}}$ cycle, Paris Sorbonne.

WARUSFEL, A. (1966) : Dictionnaire raisonné de mathématiques, Paris, Seuil. 